\title{
THE SPECIAL VALUES AT NEGATIVE INTEGERS OF DIRICHLET SERIES ASSOCIATED WITH POLYNOMIALS OF SEVERAL VARIABLES
}

\author{
MINKING EIE
}

(Communicated by William W. Adams)

ABSTRACT. Let $P(X)$ be a product of $k$ linear forms in $r$ variables $X_{1}, \ldots, X_{r}$ as given by

$$
\begin{aligned}
P\left(X_{1}, \ldots, X_{r}\right)=\prod_{j=1}^{k}\left(a_{j 1} X_{1}+\cdots+a_{j r} X_{r}+\delta_{j}\right) \\
\quad \operatorname{Re} a_{j i}>0, \operatorname{Re}\left(\delta_{j}+\sum_{i=1}^{r} a_{j i}\right)>0 .
\end{aligned}
$$

Suppose that $\beta=\left(\beta_{1}, \ldots, \beta_{r}\right)$ is an $r$-tuple of nonnegative integers. Consider the zeta function

$$
Z(P, \beta)(s)=\sum_{n_{1}=1}^{\infty} \cdots \sum_{n_{r}=1}^{\infty} n_{1}^{\beta_{1}} \cdots n_{r}^{\beta_{r}} P\left(n_{1}, \ldots, n_{r}\right)^{-s}, \quad \operatorname{Re} s>\frac{r+|\beta|}{k},
$$

where $|\beta|=\beta_{1}+\cdots+\beta_{r} . Z(P, \beta)(s)$ has an analytic continuation in the whole complex plane and it is regular at $s=0,-1,-2, \ldots,-m, \ldots$. In this paper, we shall compute the explicit values of $Z(P, \beta)(s)$ at $s=$ $0,-1,-2, \ldots,-m, \ldots$ and express them in terms of finite sums of polynomials in Bernoulli numbers.

\section{INTRODUCTION AND NOTATION}

Let $P\left(X_{1}, \ldots, X_{r}\right)$ be a polynomial of $r$ variables with positive coefficients in the real number field $\mathbf{R}$ and $\xi=\left(\xi_{1}, \ldots, \xi_{r}\right) \in \mathbf{C}^{n}$ with $\left|\xi_{i}\right|=1, \xi_{i} \neq 1$. In [1], Cassou-Nogués considered the zeta function $Z(P, \xi)(s)$ associated with $P$ and $\xi$ as follows:

$$
Z(P, \xi)(s)=\sum_{n \in \mathbf{N}^{r}} P(n)^{-s} \xi^{n}=\sum_{n_{1}=1}^{\infty} \cdots \sum_{n_{r}=1}^{\infty} P\left(n_{1}, \ldots, n_{r}\right)^{-s} \xi_{1}^{n_{1}} \cdots \xi_{r}^{n_{r}} .
$$

Received by the editors September 28, 1990 and, in revised form, January 27, 1992.

1991 Mathematics Subject Classification. Primary 11 M06.

This work was supported by the Institute of Mathematics, Academia Sinica, Taipei, Taiwan; Institute of Applied Mathematics, National Chung Cheng University; Department of Mathematics, University of Chicago; and NSF of the Republic of China (NSC 81-0208-M-001-16). 
She obtained among other results that the special values of the continuation of $Z(P, \xi)(s)$ at nonpositive integers is given by

$$
Z(P, \xi)(-m)=R\left(P^{m}\right)(\xi) .
$$

Here

$$
R\left(P^{m}\right)(T)=\sum_{n \in \mathbf{N}^{r}} P^{m}(n) T^{n}
$$

is a formal power series. Also in [1], $R\left(P^{m}\right)(\xi)$ is expressed as a finite sum of numbers of the form $\lambda\left(P^{m}\right) /\left(1-\xi_{1}\right)^{\alpha_{1}} \cdots\left(1-\xi_{r}\right)^{\alpha_{r}}$, where $\alpha_{i}$ is a nonnegative integer and $|\alpha|>0$. Obviously, the formula for $Z(P, \xi)(-m)$ did not work for the case when some $\xi_{i}=1$.

Let $Z(P)(s)=\sum_{n \in \mathbf{N}^{r}} P(n)^{-s}$. In an effort to get a similar formula for $Z(P)(-m)$, Cassou-Nogués in [2] used two different ways to investigate this problem for certain kinds of polynomials. In particular, she considered those polynomials $P$ with $P\left(X_{1}, \ldots, X_{r}\right)=C_{1} X_{1}^{m_{1}}+\cdots+C_{r} X_{r}^{m_{r}}+R\left(X_{1}, \ldots, X_{r}\right)$, where $R(X)$ is a combination of monomials $X_{1}^{\alpha_{1}} \cdots X_{r}^{\alpha_{r}}$ satisfying $\sum_{i=1}^{r} \frac{\alpha_{i}}{m_{i}} \leq$ 1. However no explicit formula for $Z(P)(-m)$ was obtained except for the special case $r=2$ and $P(X, Y)=X^{m_{1}}+Y^{m_{1}}+X^{\alpha_{1}} Y^{\alpha_{2}}$.

It is clear that the evaluation of $Z(P)(s)$ at nonpositive integers is equivalent to finding the asymptotic expansion of the series $\sum_{n \in \mathbf{N}^{r}} e^{-P(n) t}$ at $t=0$. Fractional powers may appear in the expansion and it makes the whole process more complicated.

On the other hand, Shintani [12] decomposed the Dedekind zeta function $\zeta_{\mathbf{K}}$ of a totally real number field $\mathbf{K}$ into a finite sum of zeta functions $Z(P)(s)$ as defined above with

$$
P(X)=\prod_{j=1}^{k} L_{j}(X)=\prod_{j=1}^{k}\left(a_{j 1} X_{1}+\cdots+a_{j r} X_{r}+\delta_{j}\right), \quad a_{j i}>0, \delta_{j}>0 .
$$

Let $\Gamma(s)$ be the gamma function defined by $\Gamma(s)=\int_{0}^{\infty} t^{s-1} e^{-t} d t, \operatorname{Re} s>0$. Then for $\operatorname{Re} s>r / k$, we have the integral expression

$$
Z(P)(s)[\Gamma(s)]^{k}=\int_{0}^{\infty} \cdots \int_{0}^{\infty} \frac{\left(t_{1} \cdots t_{k}\right)^{s-1} e^{-[D, T]} d t_{1} \cdots d t_{k}}{\left(e^{\left[A_{1}, T\right]}-1\right) \cdots\left(e^{\left[A_{k}, T\right]}-1\right)} .
$$

Here $[D, T]=\delta_{1} t_{1}+\cdots+\delta_{r} t_{r}$ and $\left[A_{i}, T\right]=a_{1 i} t_{1}+\cdots+a_{k j} t_{k}$.

With the above formula, Shintani was able to express $Z(P)(-m)$ in terms of coefficients of power expansions of certain functions. It turned out that these coefficients are products of Bernoulli polynomials. On the other hand, the author obtained an explicit formula for the values at nonpositive integers of a zeta function associated with a polynomial [3].

In this paper, we shall consider a more general kind of zeta function $Z(P, \beta)(s)$ defined by

$$
Z(P, \beta)(s)=\sum_{n_{1}=1}^{\infty} \cdots \sum_{n_{r}=1}^{\infty} n_{1}^{\beta_{1}} \cdots n_{r}^{\beta_{r}} P\left(n_{1}, \ldots, n_{r}\right)^{-s}, \quad \operatorname{Re} s>\frac{r+|\beta|}{k}
$$

Here $\beta=\left(\beta_{1}, \ldots, \beta_{r}\right)$ is an $r$-tuple of nonnegative integers and $P(X)=$ $\prod_{j=1}^{k} L_{j}(X), L_{j}(X)=\left(a_{j 1} X_{1}+\cdots+a_{j r} X_{r}+\delta_{j}\right), \operatorname{Re} a_{j i}>0, \operatorname{Re} L_{j}(n)>0$ for $n \in \mathbf{N}^{r}$. 
To state our main result, we let $J^{m}$ be the linear extension of $C\left[X_{1}, \ldots, X_{r}\right]$ to $C$ satisfying

$$
J^{m}\left(X_{1}^{\alpha_{1}} \cdots X_{r}^{\alpha_{r}}\right)=\prod_{i=1}^{r} \zeta\left(-\alpha_{i}\right)=(-1)^{|\alpha|} \prod_{i=1}^{r} \frac{B_{\alpha_{i}+1}}{\left(\alpha_{i}+1\right)} .
$$

Here $B_{0}, B_{1}, B_{2}, \ldots, B_{p}, \ldots$ are Bernoulli numbers defined by

$$
\frac{t}{e^{t}-1}=\sum_{p=0}^{\infty} \frac{B_{p} t^{p}}{p !}, \quad|t|<2 \pi .
$$

For $m=0$, we let $J^{0}(c)=c$. We can now give our main theorem.

Main Theorem. Let $Z(P, \beta)(s)$ be the zeta function defined as above. Then $Z(P, \beta)(s)$ is an analytic function of $s$ for $\operatorname{Re} s>(r+|\beta|) / k$ and it has an analytic continuation to the whole complex plane which is analytic except possible poles at $s=j / k, j \leq(r+|\beta|)$, where $s$ is a nonnegative integer or zero. Furthermore, the special value of $Z(P, \beta)(s)$ at nonpositive integers is given by

$$
Z(P, \beta)(-m)=\frac{1}{k} \sum_{j=1}^{k} \sum_{\left\{j_{1}, \ldots, j_{p}\right\}} J^{r-p}\left[\int_{\Delta_{j}\left(X_{j_{1}}, \ldots, X_{j_{p}}\right)} X^{\beta} P^{m}(X) d X_{j_{1}} \cdots d X_{j_{p}}\right] .
$$

Here in the second summation, $\left\{j_{1}, \ldots, j_{p}\right\}$ ranges over all subsets of $\{1, \ldots, r\}$. The domain of integration $\Delta_{j}\left(X_{j_{1}}, \ldots, X_{j_{p}}\right)$ is realized as a p-simplex in $\mathbf{R}^{p}$ defined by $L_{j}(X) \geq 0, X_{j_{1}} \leq 0, \ldots, X_{j_{p}} \leq 0$, by assuming that the coefficients of $L_{j}(X)$ are positive real numbers (see Proposition 6 for the precise meaning of the integral).

Remark. When $L_{j}(X)$ has real coefficients, then $\Delta_{j}\left(X_{j_{1}}, \ldots, X_{j_{p}}\right)$ is indeed a $p$-simplex. When $p=0$, the term $J^{r}\left[X^{\beta} P^{m}(X)\right]$ resulted easily from our calculation as we shall see.

Concerning the rationality of the values of $Z(P, \beta)(s)$ at nonpositive integers, we have the following corollary.

Corollary. Suppose that $\mathbf{k}$ is an algebraic number field over the rational number field $\mathbf{Q}$ and $\mathbf{P}$ (as given before) is a polynomial function over $\mathbf{k}$. Then for any integer $m$ with $m \geq 0, Z(P, \beta)(-m) \in \mathbf{k}$.

\section{The INTEgRAL FORMula of $Z(P, \beta)(s)$}

From now on, we use the following notation:

$$
\begin{gathered}
A_{j}=\left[a_{1 j}, \ldots, a_{k j}\right], \quad D=\left[\delta_{1}, \ldots, \delta_{k}\right], \quad T=\left[t_{1}, \ldots, t_{k}\right], \\
U=\left[u_{1}, \ldots, u_{k}\right], \quad u_{k}=1-u_{1}-\cdots-u_{k-1}, \quad d U=d u_{1} \cdots d u_{k-1}, \\
{\left[A_{j}, T\right]=\sum_{i=1}^{k} a_{i j} t_{i}, \quad\left[A_{j}, U\right]=\sum_{i=1}^{k} a_{i j} u_{i},} \\
{[D, T]=\sum_{i=1}^{k} \delta_{i} t_{i}, \quad[D, U]=\sum_{i=1}^{k} \delta_{i} u_{i},} \\
|T|=t_{1}+\cdots+t_{k}, \quad L_{j}(X)=a_{j 1} X_{1}+\cdots+a_{j r} X_{r}+\delta_{j} .
\end{gathered}
$$


Proposition 1. For $\operatorname{Re} s>|\beta|+r$, we have

$$
\begin{aligned}
Z(P, \beta)(s) \Gamma(s)^{k}=\int_{0}^{\infty} \cdots \int_{0}^{\infty} & \left(t_{1} \cdots t_{k}\right)^{s-1} e^{-[D, T]} \\
& \times \prod_{j=1}^{r}\left[\sum_{n_{j}=1}^{\infty} n_{j}^{\beta_{j}} e^{-\left[A_{j}, T\right] n_{j}}\right] d t_{1} \cdots d t_{k} .
\end{aligned}
$$

Proof. For $\operatorname{Re} s>0$ and positive integers $n_{1}, \ldots, n_{r}$, we have

$$
\begin{aligned}
& n_{1}^{\beta_{1}} \cdots n_{r}^{\beta_{r}} \prod_{j=1}^{k}\left(n+a_{j 1} n_{1}+\cdots+a_{j r} n_{r}+\delta_{j}\right)^{-s} \Gamma(s)^{k} \\
& =\int_{0}^{\infty} \cdots \int_{0}^{\infty}\left(t_{1} \cdots t_{k}\right)^{s-1} n_{1}^{\beta_{1}} \cdots n_{r}^{\beta_{r}} \\
& \quad \times \prod_{j=1}^{k} \exp \left\{-\left(a_{j 1} n_{1}+\cdots+a_{j r} n_{r}+\delta_{j}\right) t_{j}\right\} d t_{1} \cdots d t_{k} .
\end{aligned}
$$

Our assertion then follows from the monotone convergence theorem with which we can perform a term-by-term integration.

Proposition 2. For $\operatorname{Re} s>(|\beta|+r) / k$, we have

$$
\begin{aligned}
Z(P, \beta)(s) \Gamma(s)^{k}=\int_{0}^{\infty} t^{k s-1} d t \int_{E}\left(u_{1} \cdots u_{k}\right)^{s-1} e^{-[D, U] t} \\
\times \prod_{j=1}^{r}\left[\sum_{n_{j}=1}^{\infty} n_{j}^{\beta_{j}} e^{-n_{j}\left[A_{j}, U\right] t}\right] d U .
\end{aligned}
$$

Here $E$ is the standard simplex in $\mathbf{R}^{k-1}$ defined by

$$
\left\{\begin{array}{l}
u_{1}, \ldots, u_{k-1} \geq 0, \\
1-u_{1}-\cdots-u_{k-1} \geq 0 .
\end{array}\right.
$$

Proof. The proof follows from Proposition 1 and the following changing of variables which map $\left(\mathbf{R}^{+}\right)^{k}$ onto $\left(\mathbf{R}^{+}\right) \times E$ :

$$
\begin{aligned}
& t_{1}=t u_{1}, \ldots, t_{k-1}=t u_{k-1}, \quad t_{k}=t u_{k} \\
& \left(u_{1}, \ldots, u_{k-1}\right) \in E, \quad u_{k}=1-\sum_{i=1}^{k-1} u_{i} .
\end{aligned}
$$

Our assertion is true for $\operatorname{Re} s>|\beta|+r$.

Note that the function

$$
\prod_{j=1}^{r}\left[t^{\beta_{j}+1} \sum_{n_{j}=1}^{\infty} n_{j}^{\beta_{j}} e^{-\left[A_{j}, U\right] t}\right]
$$

is a rapidly decreasing function of $t$ when $0 \leq t<\infty$. Consequently, the integral on the right-hand side is convergent for $\operatorname{Re} s>(|\beta|+r) / k$. Thus the formula holds for $\operatorname{Re} s>(|\beta|+r) / k$. 
Remark. For $0<t<2 \pi$ and positive integer $p$, we have

$$
\left(\frac{d}{d t}\right)^{p} \sum_{n=1}^{\infty} e^{-n t}=\left(\frac{d}{d t}\right)^{p}\left(\frac{1}{t}+\sum_{j=1}^{\infty} \frac{B_{j} t^{j-1}}{j !}\right)
$$

With a term-by-term differentiation, we get

$$
\sum_{n=1}^{\infty} n^{p} e^{-n t}=\frac{p !}{t^{p+1}}+\sum_{j=1}^{\infty} \frac{(-1)^{p} B_{j+p+1} t^{j}}{(j+p+1) \cdot j !}
$$

Hence the series $t^{p+1} \sum_{n=1}^{\infty} n^{p} e^{-n t}$ is a regular function of $t$ at $t=0$.

3. The analyic continuation of $Z(P, \beta)(s)$

Let

$$
F(t ; U)=t^{|\beta|+r} e^{-[D, U] t} \prod_{j=1}^{r}\left\{\sum_{n_{j}=1}^{\infty} n_{j}^{\beta_{j}} e^{-n_{j}\left[A_{j}, U\right] t}\right\}
$$

and

$$
I(s, t)=\frac{1}{\Gamma(s)^{k-1}} \int_{E}\left(u_{1} \cdots u_{k}\right)^{s-1} F(t ; U) d u_{1} \cdots d u_{k-1} .
$$

Then we can rewrite $Z(P, \beta)(s) \Gamma(s)$ as

$$
Z(P, \beta)(s) \Gamma(s)=\int_{0}^{\infty} t^{k s-|\beta|-r-1} I(s, t) d t .
$$

$F(t ; U)$ is a continuous function in $u_{1}, \ldots, u_{k-1}$ and a rapidly decreasing function in $t$, and the measure

$$
\frac{\Gamma(k s)\left(u_{1} \cdots u_{k}\right)^{s-1} d u_{1} \cdots d u_{k-1}}{\Gamma(s)^{k}}
$$

is a bounded measure on the standard simplex $E$ (see the following Remark). Thus $I(s, t)$ is a holomorphic function in $s$ and a rapidly decreasing function for $0 \leq t<\infty$. Hence $Z(P, \beta)(s) \Gamma(s)$ has only possible simple poles at $s=j / k, j \leq r+|\beta|, j \in \mathbf{Z}$, with $\mathbf{Z}$ being the set of integers.

Remark. The measure $\Gamma(k s)\left(u_{1} \cdots u_{k}\right)^{s-1} d u_{1} \cdots d u_{k-1} / \Gamma(s)^{k}$ is the wellknown classical Dirichlet measure. It is well defined for $\operatorname{Re} s>0$ and has its analytic continuation for all $s$.

Proposition 3. Let the power expansion of $F(t ; U)$ at $t=0$ be $\sum_{i=0}^{\infty} t^{i} F(U)$. Then

$$
Z(P, \beta)(-m)=\frac{(-1)^{m} m !}{k} G_{k m+|\beta|+r}(-m),
$$

with

$$
G_{i}(s)=\frac{1}{\Gamma(s)^{k-1}} \int_{E}\left(u_{1} \cdots u_{k}\right)^{s-1} F_{i}(U) d u_{1} \cdots d u_{k-1}, \quad \operatorname{Re} s>0
$$


Proof.

$$
\begin{aligned}
Z(P, \beta)(s) \Gamma(s) & =\int_{0}^{1} t^{k s-|\beta|-r-1} I(s, t) d t+\int_{1}^{\infty} t^{k s-|\beta|-r-1} I(s, t) d t \\
& =\int_{0}^{1} t^{k s-|\beta|-r-1}\left\{\sum_{i=0}^{\infty} t^{i} G_{i}(s)\right\} d t+\int_{1}^{\infty} t^{k s-|\beta|-r-1} I(s, t) d t \\
& =\sum_{i=0}^{\infty} \frac{G_{i}(s)}{k s-|\beta|-r+i}+\int_{1}^{\infty} t^{k s-|\beta|-r-1} I(s, t) d t
\end{aligned}
$$

The integral on the right-hand side is convergent for any $s$, so the right-hand side is a meromorphic function in $s$, which gives the analytic continuation of $Z(P, \beta)(s) \Gamma(s)$. For $s=-m$, the residue of the right-hand side is $\frac{1}{k} G_{k m+|\beta|+r}(-m)$. But as $\Gamma(s)$ has a simple pole at $s=-m$, it forces $Z(P, \beta)(s)$ to be regular at $s=-m$ and

$$
\frac{(-1)^{m}}{m !} Z(P, \beta)(-m)=\frac{1}{k} G_{k m+|\beta|+r}(-m) .
$$

It follows that

$$
Z(P, \beta)(-m)=\frac{(-1)^{m} m !}{k} G_{k m+|\beta|+r}(-m)
$$

\section{THE EXPLICIT FORMULA}

Applying the formula

$$
\sum_{n=1}^{\infty} n^{p} e^{-n t}=\frac{p !}{t^{p+1}}+\sum_{j=1}^{\infty} \frac{(-1)^{p} B_{j+p+1} t^{j}}{(j+p+1) \cdot j !}, \quad 0<t<2 \pi,
$$

we get the following power series expansions for $0 \leq t<2 \pi$ :

$$
\begin{aligned}
t^{\beta_{1}+1} & \sum_{n_{1}=1}^{\infty} n_{1}^{\beta_{1}} e^{-n_{1}\left[A_{1}, U\right] t} \\
& =\frac{\beta_{1} !}{\left[A_{1}, U\right]^{\beta_{1}+1}}+\sum_{j=0}^{\infty} \frac{(-1)^{\beta_{1}} B_{j+\beta_{1}+1}\left[A_{1}, U\right]^{j} t^{j+\beta_{1}+1}}{\left(j+\beta_{1}+1\right) \cdot j !}
\end{aligned}
$$

$\left(\Sigma_{r}\right)$

$$
t^{\beta_{r}+1} \sum_{n_{r}=1}^{\infty} n_{1}^{\beta_{r}} e^{-n_{r}\left[A_{r}, U\right] t}
$$

$$
=\frac{\beta_{r} !}{\left[A_{r}, U\right]^{\beta_{r}+1}}+\sum_{j=0}^{\infty} \frac{(-1)^{\beta_{r}} B_{j+\beta_{r}+1}\left[A_{r}, U\right]^{j} t^{j+\beta_{r}+1}}{\left(j+\beta_{r}+1\right) \cdot j !},
$$

$\left(\Sigma_{r+1}\right)$

$$
e^{-[D, U] t}=\sum_{j=0}^{\infty} \frac{(-1)^{j}[D, U]^{j} t^{j}}{j !} .
$$

$G_{k m+|\beta|+r}(s)$ is an integration over $E$ of the coefficient of $t^{k m+|\beta|+r}$ in the product of series $\left(\Sigma_{1}\right), \ldots,\left(\Sigma_{r}\right),\left(\Sigma_{r+1}\right)$. So it is a finite linear combination of 
integrals of the form

(4)

$H_{\alpha}(s)=\frac{1}{\Gamma(s)^{k-1}} \int_{E}\left(u_{1} \cdots u_{k}\right)^{s-1}\left[A_{1}, U\right]^{\alpha_{1}} \cdots\left[A_{r}, U\right]^{\alpha_{r}}[D, U]^{\alpha_{r+1}} d u_{1} \cdots d u_{k-1}$

with $\alpha_{i} \geq 0$ or $\alpha_{i}=-\beta_{i}-1 \quad(i=1, \ldots, r), \alpha_{r+1} \geq 0$, and $\sum_{i=1}^{r+1}\left|\alpha_{i}\right|=$ $\mathrm{km}$. More precisely, the general terms in $G_{k m+|\beta|+r}(s)$ can be divided into the following two types of integrals:

$$
\sum_{|\alpha|=k m}(-1)^{|\beta|}\left[\prod_{i=1}^{r} \frac{B_{\alpha_{i}+\beta_{i}+1}}{\left(\alpha_{i}+\beta_{i}+1\right) \alpha_{i} !}\right] \frac{(-1)^{\alpha_{r+1}}}{\alpha_{r+1} !} H_{\alpha}(s),
$$

where $\alpha=\left(\alpha_{1}, \ldots, \alpha_{r}, \alpha_{r+1}\right)$ is an $(r+1)$-tuple of nonnegative integers, and

$$
\begin{aligned}
& \sum_{\alpha} \beta_{1} ! \cdots \beta_{q} !(-1)^{\beta_{q+1}+\cdots+\beta_{r}}\left[\prod_{i=q+1}^{r} \frac{B_{\alpha_{i}+\beta_{i}+1}}{\left(\alpha_{i}+\beta_{i}+1\right) \alpha_{i} !}\right] \frac{(-1)^{\alpha_{r+1}}}{\alpha_{r+1} !} \\
& \quad \times \frac{1}{\Gamma(s)^{k-1}} \int_{E} \frac{\left(u_{1} \cdots u_{k}\right)^{s-1}\left[A_{q+1}, U\right]^{\alpha_{q+1}} \cdots\left[A_{r}, U\right]^{\alpha_{r}}[D, U]^{\alpha_{r+1}} d U}{\left[A_{1}, U\right]^{\beta_{1}+1} \cdots\left[A_{q}, U\right]^{\beta_{q}+1}}
\end{aligned}
$$

Here in the summation, $\alpha=\left(\alpha_{q+1}, \ldots, \alpha_{r}, \alpha_{r+1}\right)$ ranges over all $(r-q+1)$ tuples of integers satisfying $|\alpha|=k m+\beta_{1}+\cdots+\beta_{q}+q$.

In our propositions which follow, we shall devote ourselves to the evaluation of $H_{\alpha}(s)$ at $s=-m$ for various $\alpha$ 's appearing in the summation of $G_{k l m+|\beta|+r}$ and combine them to yield the Bernoulli numbers associated with polynomials as given in the main theorem.

Proposition 4. Let $H_{\alpha}(s)$ be the integral as defined in (4). Then

$$
\begin{aligned}
& \frac{(-1)^{m} m !}{k} \sum_{\substack{0 \leq \alpha_{1}, \ldots, \alpha_{r+1} \leq m ;|\alpha|=k m \\
=J^{r}\left[X^{\beta} P^{m}(X)\right] .}}(-1)^{|\beta|}\left[\prod_{i=1}^{r} \frac{B_{\alpha_{i}+\beta_{i}+1}}{\left(\alpha_{i}+\beta_{i}+1\right) \alpha_{i} !}\right] \frac{(-1)^{\alpha_{r+1}}}{\alpha_{r+1} !} H_{\alpha}(-m) \\
& \quad
\end{aligned}
$$

Proof.

$$
\begin{aligned}
H_{\alpha}(s)= & \prod_{i=1}^{r} \sum_{\alpha_{1 i}+\cdots+\alpha_{k i}=\alpha_{i}} \frac{\alpha_{i} !\left(a_{1 i}\right)^{\alpha_{1 i} \cdots\left(a_{k i}\right)^{\alpha_{k i}}}}{\alpha_{l i} ! \cdots \alpha_{k i} !} \\
& \times \sum_{\alpha_{1 r+1}+\cdots+\alpha_{k r+1}=\alpha_{r+1}} \frac{\alpha_{r+1} !\left(\delta_{1}\right)^{\alpha_{1 r+1}} \cdots\left(\delta_{k}\right)^{\alpha_{k r+1}}}{\alpha_{1 r+1} ! \cdots \alpha_{k r+1} !} A_{\alpha}(s)
\end{aligned}
$$

with

$$
A_{\alpha}(s)=\frac{1}{\Gamma(s)^{k-1}} \int_{E} u_{1}^{s+b_{1}-1} \cdots u_{k}^{s+b_{k}-1} d u_{1} \cdots d u_{k-1} .
$$

Here $b_{1}=\alpha_{11}+\alpha_{12}+\cdots+\alpha_{1 r+1}, \ldots, b_{k}=\alpha_{k 1}+\alpha_{k 2}+\cdots+\alpha_{k r+1}$. Note that

$$
A_{\alpha}(s)=\frac{\Gamma\left(s+b_{1}\right) \cdots \Gamma\left(s+b_{k}\right)}{\Gamma(s)^{k-1} \Gamma(k s+|\alpha|)}
$$

and

$$
A_{\alpha}(-m)=\left\{\begin{array}{l}
(-1)^{(k-1) m} \frac{(m !)^{k-1} k(k m-|\alpha|) !}{\left(m-b_{1}\right) ! \cdots\left(m-b_{k}\right) !} \\
0 \text { otherwise. }
\end{array}\right.
$$


In our cases, we have $b_{1}+\cdots+b_{k}=|\alpha|=k m$. Hence it forces $b_{1}=b_{2}=$ $\cdots=b_{k}=m$ when $A_{\alpha}(-m)$ is not zero. For such cases, we have $A_{\alpha}(-m)=$ $(-1)^{k m-m}(m !)^{k-1}$. Our assertion then follows from the multinomial expansion of $X^{\beta} P^{m}(X)$ and our definition of $J^{r}\left[X^{\beta} P^{m}(X)\right]$.

For the remaining cases, we have to find the value of $s=-m$ of

$$
\frac{1}{\Gamma(s)^{k-1}} \int_{E} \frac{\left(u_{1} \cdots u_{k}\right)^{s-1}\left[A_{q+1}, U\right]^{\alpha_{q+1}} \cdots\left[A_{r}, U\right]^{\alpha_{r}}[D, U]^{\alpha_{r+1}} d U}{\left[A_{1}, U\right]^{\beta_{1}+1} \cdots\left[A_{q}, U\right]^{\beta_{q}+1}}
$$

which is a linear combination of

$$
G(\beta ; b)(s)=\frac{1}{\Gamma(s)^{k-1}} \int_{E} \frac{u_{1}^{s+b_{1}-1} \cdots u_{k}^{s+b_{k}-1} d U}{\left[A_{1}, U\right]^{\beta_{1}+1} \cdots\left[A_{q}, U\right]^{\beta_{q}+1}} \quad(1 \leq q \leq r),
$$

with $b_{1}+\cdots+b_{k}=\alpha_{q+1}+\cdots+\alpha_{r+1}=k m+q+\beta_{1}+\cdots+\beta_{q}$. First we observe the following facts concerning $G(\beta ; b)$.

(1) The integral in $G(\beta ; b)(s)$ as a function of $s$ has a pole at $s=-m$ of order at most $k-1$ arising from integration around the vertices of $E$.

(2) If there are more than one $b_{j}$ in $\left\{b_{1}, \ldots, b_{k}\right\}$ which are greater than $m$, then the order of the pole at $s=-m$ for the integral will be less than $k-1$. By considering the fact that $\Gamma(s)^{k-1}$ always has a pole at $s=-m$ of order $k-1$, we conclude that $G(\beta ; b)(-m)=0$.

With the above considerations, it suffices to consider the case when $b_{j} \geq$ $m+1$ for exactly one $j$. In the following proposition, we suppose that $0 \leq$ $b_{1}, \ldots, b_{k-1} \leq m$ and $b_{k} \geq m+1$. To determine the explicit value of $G(\beta ; b)(-m)$, we need the usual notation of partial differential operators. Let

$$
D_{1}=\frac{\partial}{\partial u_{1}}, \ldots, D_{k-1}=\frac{\partial}{\partial u_{k-1}}, \quad D^{\alpha}=D_{1}^{\alpha_{1}} \cdots D_{k-1}^{\alpha_{k-1}} .
$$

Also $\alpha !=\alpha_{1} ! \cdots \alpha_{k-1}$ ! if $\alpha=\left(\alpha_{1}, \ldots, \alpha_{k-1}\right)$.

Proposition 5. For $b=\left(b_{1}, \ldots, b_{k}\right)$ with $|b|=k m+|\beta|+q, 0 \leq b_{1}, \ldots, b_{k-1}$ $\leq m$, and $b_{k} \geq m+1$, we have

$$
\begin{aligned}
G(\beta ; b)(-m)=\sum_{\alpha_{1}+\cdots+\alpha_{q+1}=\mathbf{m}-\mathbf{b}} & {\left[\prod_{j=1}^{k-1} \prod_{i=1}^{q}\left(\frac{a_{k i}-a_{j i}}{a_{k i}}\right)^{\alpha_{j i}} \frac{1}{\alpha_{j i} !}\right] } \\
& \times \prod_{i=1}^{q} \frac{\left(\beta_{i}+\left|\alpha_{i}\right|\right) !}{\beta_{i} ! a_{k i}^{\beta_{i}+1}} \frac{(-1)^{\left|\alpha_{q+1}\right|}\left(b_{k}-m-1\right) !}{\left(b_{k}-m-1-\left|\alpha_{q+1}\right|\right) !} .
\end{aligned}
$$

Here $\alpha_{i}=\left(\alpha_{1 i}, \ldots, \alpha_{k-1, i}\right) \quad(i=1, \ldots, q+1)$ and $\mathbf{m}-\mathbf{b}=\left(m-b_{1}, \ldots\right.$, $\left.m-b_{k-1}\right)$.

Proof. When $0 \leq b_{1}, \ldots, b_{k-1} \leq m$ and $b_{k} \geq m+1$, the integral in $G(\beta ; b)(s)$ has a pole of order $k-1$ at $s=-m$ arising from integration around the vertex $\left(u_{1}, \ldots, u_{k-1}, u_{k}\right)=(0, \ldots, 0,1)$. Also the integrations around other vertices result in poles with order less than $k-1$. By the regularity of the 
integral [3]

$$
\begin{aligned}
G(\beta ; b)(-m)= & \text { the coefficient of } u_{1}^{m-b_{1}} \cdots u_{k-1}^{m-b_{k-1}} \text { of the function } \\
& {\left[A_{1}, U\right]^{-\beta_{1}-1} \cdots\left[A_{q}, U\right]^{-\beta_{q}-1}\left(1-u_{1}-\cdots-u_{k-1}\right)^{b_{k}-m-1} } \\
& \text { expanded at } u_{1}=\cdots=u_{k-1}=0 \\
= & \frac{1}{(\mathbf{m}-\mathbf{b}) !} D^{\mathbf{m}-\mathbf{b}}\left\{\left[A_{1}, U\right]^{-\beta_{1}-1} \cdots\left[A_{q}, U\right]^{-\beta_{q}-1}\right. \\
& \left.\quad \times\left(1-u_{1}-\cdots-u_{k-1}\right)^{b_{k}-m-1}\right\}_{u_{1}=\cdots=u_{k-1}=0} .
\end{aligned}
$$

By Leibniz's rule for partial differential operators, we have

$$
\begin{gathered}
\frac{1}{(\mathbf{m}-\mathbf{b}) !} D^{\mathbf{m}-\mathbf{b}}\left[\left[A_{1}, U\right]^{-\beta_{1}-1} \cdots\left[A_{q}, U\right]^{-\beta_{q}-1}\left(1-u_{1}-\cdots-u_{k-1}\right)^{b_{k}-m-1}\right] \\
=\sum_{\alpha_{1}+\cdots+\alpha_{q+1}=\mathbf{m}-\mathbf{b}} \frac{1}{\alpha_{1} ! \cdots \alpha_{q+1} !}\left[\prod_{i=1}^{q} D^{\alpha_{i}}\left[A_{i}, U\right]^{-\beta_{i}-1}\right] \\
\times D^{\alpha_{q+1}}\left(1-u_{1}-\cdots-u_{k-1}\right)^{b_{k}-m-1} .
\end{gathered}
$$

Replace $\left[A_{j}, U\right]$ by $a_{1 j} u_{1}+\cdots+a_{k-1, j} u_{k-1}+a_{k j}\left(1-u_{1}-\cdots-u_{k-1}\right)$. Then an elementary calculation yields our values for $G(\beta ; b)(-m)$.

The following lemma is needed in our proof of the next proposition.

Lemma. Let $f$ be a continuous function on $[0, \infty), b_{1}, \ldots, b_{q}$ nonnegative integers, and $E(h)$ the simplex defined by

$$
0 \leq u_{1}+\cdots+u_{q} \leq h, \quad u_{1}, \ldots, u_{q} \geq 0, h>0 .
$$

Then

$$
\begin{aligned}
\int_{E(h)} & \left(u_{1}^{b_{1}} \cdots u_{q}^{b_{q}}\right) f\left(u_{1}+\cdots+u_{q}\right) d u_{1} \cdots d u_{q} \\
= & \frac{b_{1} ! \cdots b_{q} !}{\left(b_{1}+\cdots+b_{q}+q-1\right) !} \int_{0}^{h} f(t) t^{b_{1}+\cdots+b_{q}+q-1} d t .
\end{aligned}
$$

Proof. The lemma follows from a change of variables and the definition of a $\beta$-function of several variables.

Proposition 6. Let $R_{j}(X)=\sum_{i=q+1}^{r} a_{j i} X_{i}+\delta_{j}(j=1, \ldots, k)$. Then

$$
\begin{aligned}
\int_{\Delta_{k}\left(X, \ldots, X_{q}\right)} X^{\beta} P^{m}(X) d X_{1} \cdots d X_{q} \\
=(m !)^{k} \sum_{b} \prod_{j=1}^{k} \frac{R_{j}^{b_{j}}(X)}{b_{j} !} \sum_{\alpha_{1}+\cdots+\alpha_{q+1}=\mathbf{m}-\mathbf{b}} \prod_{j=1}^{k-1} \prod_{i=1}^{q}\left(\frac{a_{k i}-a_{j i}}{a_{k i}}\right)^{\alpha_{j i}} \\
\quad \times \prod_{i=1}^{q} \frac{\left(\beta_{i}+\mid \alpha_{i}\right) !}{\beta_{i} ! a_{k i}^{\beta_{i}+1}}(-1)^{\left|\alpha_{q+1}\right|+|\beta|+q}\left(b_{k}-m-1\right) !
\end{aligned}
$$

Here $b$ ranges over all $k$-tuple nonnegative integers $\left(b_{1}, \ldots, b_{k}\right)$ satisfying $0 \leq b_{1}, \ldots, b_{k-1} \leq m,|b|=k m+|\beta|+1$, and $b_{k} \geq m+1$. Also $\alpha_{j}=$ $\left(\alpha_{1 j}, \ldots, \alpha_{k-1, j}\right) \quad(j=1, \ldots, q+1)$. 
Proof. Rewrite the integral as

$$
\begin{aligned}
& \sum_{0 \leq b_{1}, \ldots, b_{k-1} \leq m} \prod_{i=q+1}^{r} X_{i}^{\beta_{i}} \prod_{j=1}^{k-1} \frac{m !}{\left(m-b_{j}\right) ! b_{j} !} R_{j}^{b_{j}}(X) \\
& \quad \times \int_{\Delta_{k}\left(X, \ldots, X_{q}\right)} \prod_{i=1}^{q} X_{i}^{\beta_{i}} \prod_{j=1}^{k-1}\left(a_{j 1} X_{1}+\cdots+a_{j q} X_{q}\right)^{m-b_{j}} L_{k}(X) d X_{1} \cdots d X_{q} .
\end{aligned}
$$

Now let $X_{i}=-u_{i} R_{k}(X) / a_{k i}(i=1, \ldots, q)$. Then $\Delta_{k}\left(X_{1}, \ldots, X_{q}\right)$ is transformed into the standard $q$-simplex $E^{q}$ defined by

$$
E^{q}: u_{1} \geq 0, \ldots, u_{q} \geq 0,1-u_{1}-\cdots-u_{q} \geq 0 .
$$

Consequently, we have

$$
\begin{aligned}
\int_{\Delta_{k}\left(X, \ldots, X_{q}\right)} \prod_{i=1}^{q} X_{i}^{\beta_{i}} \prod_{j=1}^{k-1}\left(a_{j 1} X_{1}+\cdots+a_{j q} X_{q}\right)^{m-b_{j}} L_{k}(X) d X_{1} \cdots d X_{q} \\
=\prod_{i=1}^{q}\left(-\frac{1}{a_{k j}}\right)^{\beta_{j}+1}\left[R_{k}(X)\right]^{b_{k}} \int_{E^{q}} \prod_{i=1}^{q} u_{i}^{\beta_{i}} \prod_{j=1}^{k-1}\left(-\frac{a_{j 1} u_{1}}{a_{k 1}}-\cdots-\frac{a_{j q} u_{q}}{a_{k q}}\right)^{m-b_{j}} \\
\quad \times\left(1-u_{1}-\cdots-u_{q}\right)^{m} d u_{1} \cdots d u_{q} .
\end{aligned}
$$

Note that

$$
\begin{aligned}
& \left(-\frac{a_{j 1}}{a_{k 1}} u_{1}-\cdots-\frac{a_{j q}}{a_{k q}} u_{q}\right)^{m-b_{j}} \\
& =\left[\left(\frac{a_{k 1}-a_{j 1}}{a_{k 1}} u_{1}\right)+\cdots+\left(\frac{a_{k q}-a_{j q}}{a_{k q}} u_{q}\right)-\left(u_{1}-\cdots-u_{q}\right)\right]^{m-b_{j}} \\
& \quad=\sum_{\alpha_{j 1}+\cdots+\alpha_{j, q+1}=m-b_{j}} \frac{\left(m-b_{j}\right) !}{\alpha_{j 1} ! \cdots \alpha_{j, q+1} !}\left(\frac{a_{k 1}-a_{k 1}}{a_{k 1}} u_{1}\right)^{\alpha_{j 1}} \cdots\left(\frac{a_{k q}-a_{j q}}{a_{k q}} u_{q}\right)^{\alpha_{j q}} \\
& \times(-1)^{\alpha_{j, q+1}}\left(u_{1}+\cdots+u_{q}\right)^{\alpha_{j, q+1}}
\end{aligned}
$$

Hence we have

$$
\begin{gathered}
\int_{E^{q}} \prod_{i=1}^{q} u_{i}^{\beta_{i}} \prod_{j=1}^{k-1}\left(-\frac{a_{j 1} u_{1}}{a_{k 1}}-\cdots-\frac{a_{j q} u_{q}}{a_{k q}}\right)^{m-b_{j}}\left(1-u_{1}-\cdots-u_{q}\right)^{m} d u_{1} \cdots d u_{q} \\
=\sum_{\alpha_{1}+\cdots+\alpha_{q+1}=m-b}\left(\prod_{i=1}^{q} \prod_{j=1}^{k-1}\left(\frac{a_{k i}-a_{j i}}{a_{k i}}\right)^{\alpha_{j i}} \cdot \frac{1}{a_{j i} !}\right)(-1)^{\left|\alpha_{q+1}\right|} \\
\times \int_{E^{q}} u_{1}^{\beta_{1}+\left|\alpha_{1}\right|} \cdots u_{q}^{\beta_{q}+\left|\alpha_{q}\right|}\left(1-u_{1}-\cdots-u_{q}\right)^{m} \\
\times\left(u_{1}+\cdots+u_{q}\right)^{\left|\alpha_{q+1}\right|} d u_{1} \cdots d u_{q} .
\end{gathered}
$$

By our lemma, the integral is equal to

$$
\frac{\left[\prod_{i=1}^{q}\left(\beta_{i}+\left|\alpha_{i}\right|\right) !\right] m !\left(b_{k}-m-1\right) !}{b_{k} !}
$$

Combining the previous propositions, we get the following proposition, which is precisely what we needed. 
Proposition 7. With notation as above, we have

$$
\begin{aligned}
(-1)^{m} m ! & \sum_{\alpha} \beta_{1} ! \cdots \beta_{q} !(-1)^{\beta_{q+1}+\cdots+\beta_{r}}\left[\prod_{i=q+1}^{r} \frac{B_{\alpha_{i}}+\beta_{i}+1}{\alpha_{i}+\beta_{i}+1}\right] \frac{(-1)^{\alpha_{r+1}}}{\alpha_{r+1} !} \\
& \times \sum_{b} \prod_{j=1}^{q} \prod_{i=q+1}^{r+1} \cdot \frac{1}{\alpha_{j i} !} G(\beta ; b)(-m) \\
= & J^{r-q}\left[\prod_{j=1}^{k} \int_{\Delta_{k}\left(X, \ldots, X_{q}\right)} X^{\beta} P^{m}(X) d X_{1} \cdots d X_{q}\right] .
\end{aligned}
$$

Here $\alpha$ ranges over all nonnegative integers $\left(\alpha_{q+1}, \ldots, \alpha_{r}, \alpha_{r+1}\right)$ such that $|\alpha|=k m+|\beta|+q$ in the first summation, and the relation between $b$ and $\alpha$ is

$$
\begin{aligned}
& b=\left(b_{1}, \ldots, b_{k}\right)=\left(\sum_{i=q+1}^{r} \alpha_{1 i}, \ldots, \sum_{i=q+1}^{r} \alpha_{k i}\right) \\
& \text { if } \alpha_{i}=\alpha_{1 i}+\cdots+\alpha_{k i}(i=q+1, \ldots, r+1) .
\end{aligned}
$$

\section{REFERENCES}

1. P. Cassou-Nogués, Valeurs aux entiers negatifs des series de Dirichlet associees a un polynome. I, J. Number Theory 14 (1982), 32-64.

2. _ Series de Dirichlet et integrales associees a un polynome a deux indeterminees, J. Number Theory 23 (1986), 1-54.

3. Minking Eie, On a Dirichlet series associated with a polynomial, Proc. Amer. Math. Soc. 110 (1990), 583-590.

4. I. M. Gel'fand and G. E. Shilov, Generalized function, vol. 1, Academic Press, New York and London, 1964.

5. D. Kramer, On the values at integers of the Dedekind zeta function of a real quadratic field, Trans. Amer. Math. Soc. 299 (1987), 59-79.

6. A. Kurihara, On the values at non-positive integers of Siegel's zeta functions of q-anisotropic quadratic forms with signature $(1, n-1)$, J. Fac. Sci. Univ. Tokyo Sect. IA Math. 28 (1981), 567-584.

7. P. Sargos, Prolongement meromorphe des series de Dirichlet associees a des fractions rationelles a plusieurs variables, Ann. Inst. Fourier 34 (1984).

8. I. Satake, Special values of zeta functions associated with self dual homogeneous cones, manuscript, 1981.

9. I. Satake and S. Ogata, Zeta functions associated to cones and their special values, manuscript, 1987.

10. M. Sato and T. Shintani, On zeta functions associated with prehomogeneous vector spaces, Ann. of Math. (2) 100 (1974), 131-170.

11. T. Shintani, Zeta-functions associated with the vector of quadratic forms, J. Fac. Sci. Univ. Tokyo Sect. IA Math. 22 (1975), 25-65.

12. __ On evaluation of zeta functions of totally real algebraic number fields at non-positive integers, J. Fac. Sci. Univ. Tokyo Sect. IA Math. 23 (1976), 393-417.

Institute of Applied Mathematics, National Chung Cheng University, Ming-Hsiung Chia-Yi, Taiwan, Republic of China

E-mail address: mkeie@math.ccu.edu.tw 